Contribution to fusion research from IAEA coordinated research projects and joint experiments

This content has been downloaded from IOPscience. Please scroll down to see the full text.

View the table of contents for this issue, or go to the journal homepage for more

Download details:

IP Address: 134.94.244.17

This content was downloaded on 16/09/2015 at 08:02

Please note that terms and conditions apply. 


\title{
Contribution to fusion research from IAEA coordinated research projects and joint experiments
}

\author{
M. Gryaznevich ${ }^{1,6,7}$, G. Van Oost ${ }^{2}$, J. Stöckel ${ }^{3}$, R. Kamendje ${ }^{5}$, \\ B.N. Kuteev ${ }^{4}$, A. Melnikov ${ }^{4,10}$, T. Popov ${ }^{8}$, V. Svoboda ${ }^{9}$ and \\ The IAEA CRP Teams \\ ${ }^{1}$ Tokamak Energy Ltd, Culham Science Centre, Abingdon, UK \\ ${ }^{2}$ Department of Applied Physics, Ghent University, Belgium \\ ${ }^{3}$ Institute of Plasma Physics ASCR, Prague, Czech Republic \\ ${ }^{4}$ National Research Centre 'Kurchatov Institute', 123182, Moscow, Russia \\ ${ }^{5}$ IAEA NAPC Physics Section, Vienna, Austria \\ ${ }^{6}$ Technical University of Denmark, Fysikvej, Denmark \\ ${ }^{7}$ Imperial College, London, UK \\ ${ }^{8}$ Sophia University, Sophia, Bulgaria \\ ${ }^{9}$ Technical University of Prague, Czech Republic \\ ${ }^{10}$ National Research Nuclear University MEPhI, 115409, Moscow, Russia \\ E-mail: Mikhail.gryaznevich@tokamakenergy.co.uk
}

Received 3 December 2014, revised 27 March 2015

Accepted for publication 7 April 2015

Published 30 July 2015

\section{Abstract}

The paper presents objectives and activities of IAEA Coordinated Research Projects 'Conceptual development of steady-state compact fusion neutron sources' and 'Utilisation of a network of small magnetic confinement fusion devices for mainstream fusion research'. The background and main projects of the CRP on FNS are described in detail, as this is a new activity at IAEA. Recent activities of the second CRP, which continues activities of previous CRPs, are overviewed.

Keywords: IAEA CRP, IAEA JE, small tokamaks, fusion neutron source

(Some figures may appear in colour only in the online journal)

\section{Introduction}

IAEA Coordinated Research Projects (CRPs) on 'Conceptual development of steady-state compact fusion neutron sources (CFNSs), 2012-2016', and 'Utilisation of a network of small magnetic confinement fusion devices for mainstream fusion research, 2011-2015', continue to contribute to the mainstream Fusion Research. These CRPs join participants from 18 IAEA member states, who perform and present results of individual and coordinated research and results obtained at annual IAEA joint experiments (JEs), at regular biannual CRP Research Coordinating Meetings, at international conferences and in journal publications. These activities also create platform for building long term relationships between scientists from developing and developed countries. This helps in encouraging further bilateral and/or multilateral collaborations among institutions in various member states in the field of fusion science and technology.

The paper presents the background and overviews main projects of the CRP on FNS in detail, as this is a new activity at IAEA and this is the first presentation of its goals and first results. The second CRP is a continuation of previous activities at IAEA on small fusion devices. Recent results obtained by participating Institutions are presented, including results for the latest IAEA JE on COMPASS and GOLEM in Prague, November 2014. Educational and training activities within the CRP are discussed.

\section{CRP F1.30.15 'Conceptual development of steady-state compact fusion neutron sources'}

Both the fusion and fission energy sectors need the availability of additional neutron sources to aid in their development and solve their fuel cycle problems. Moreover, the availability of neutron sources with high intensity and high flux will broaden, even qualitatively, the scope of basic research and technology activities including neutron scattering, neutron dipole moment measurements, materials development, transmutation and components testing for fusion and fission applications. Reasonable extrapolations from current machines indicate that magnetic fusion neutron sources, with a potential of providing intensities up to $10^{20}$ neutrons $\mathrm{s}^{-1}$ and fluxes higher than 
$10^{15} \mathrm{n} \mathrm{cm}^{-2} \mathrm{~s}^{-1}$, are a near term possibility. The urgent need for a CFNS has been recently confirmed in the European fusion roadmap [1].

The application of neutrons is one of the few remaining areas in which new scientific breakthroughs are limited by the available source intensity. Neutrons for commercial and research applications are currently produced in nuclear reactors, spallation sources, dense plasma focus devices and by using radioactive isotopes.

The high flux research reactor at Institute Laue-Langevin (Grenoble, France) produces neutrons with power up to $1.5 \mathrm{MW}\left(10^{18}\right.$ neutrons $\left.\mathrm{s}^{-1}\right)$. The spallation source at ORNL (USA) is less intense $\sim 0.3 \mathrm{MW}\left(10^{17}\right.$ neutrons $\left.\mathrm{s}^{-1}\right)$. These neutron sources have already reached the level at which the increase in the neutron output is limited by the engineering constraints and existing technologies. Lower-power neutron sources with source strength up to $10^{14}$ neutrons $\mathrm{s}^{-1}$, such as the low energy neutron source (LENS) facility now under construction at Indiana University in the USA, require a local capability in accelerator technology and engineering, and a capital investment in the range of US \$10-30 M, with operating costs in the range of $10-15 \%$ of the capital cost. Mediumpower pulsed neutron sources (e.g. ISIS in the UK and the Lujan Center in the USA) with source strengths up to $10^{16}$ neutrons $\mathrm{s}^{-1}$ require substantial infrastructure to design and build. Costs associated with a medium-power facility are an order of magnitude higher than that of a low-power facility. All other neutron sources are several orders of magnitude lower in neutron fluxes. For example, commercially produced turnkey neutron sources are readily available with source strengths up to $10^{13}$ neutrons $\mathrm{s}^{-1}$.

Fusion neutron sources are rather prolific in neutrons: the European tokamak JET already produced (in 1997) $5 \times$ $10^{18}$ neutrons $\mathrm{s}^{-1}$ in pulsed operations, while the number will reach $2 \times 10^{20}$ neutrons $\mathrm{s}^{-1}$ with ITER. Steady-state fusion neutron sources at any power level will open opportunities for many applications for which the current sources may not be adequate. The development of fusion nuclear technologies requires steady-state devices with high output of high energy neutrons, complementary to International Fusion Materials Irradiation Facility (IFMIF) and existing low-power neutron sources. In particular, the engineering design of a demonstration fusion power plant will require component test facilities with $14 \mathrm{MeV}$ neutrons to test and qualify different components and modules.

At present, mainstream fusion research is aimed at energy production. However, it is well known (i.e. [18], figure 23) that in a tokamak the optimal conditions for energy production are different from those for maximum neutron production. Emphasis on 'fusion for neutrons' will, therefore, broaden the scope of fusion research, in particular in exploring plasma modes that are more suited for neutron production; the motivation is provided by the large number of possible applications of copious fusion neutrons. High energy fusion neutrons are valuable for a range of technological applications such as manufacturing medical isotopes and testing materials and components for use in future fusion reactors; fusion neutrons could also greatly assist fission reactors (current and future designs) in a variety of ways. In addition, a wide variety of other applications for neutrons exist including (i) the detection of specific elements or isotopes in complex environments, (ii) radiotherapy, (iii) the alteration of electrical, optical or mechanical properties of materials and other material studies, (iv) the production of hydrogen (via high-temperature electrolysis), (v) the production of tritium (scarce due to its short half-life), and (vi) many other non-electric applications of fusion. These possible commercial non-electrical applications have been analysed in detail in the FESAC and ARIES (USA) studies and other reports [20-24]. But the most obvious non-electric application of a fusion neutron source is to aid the presently expanding nuclear energy industry, which is rapidly both exhausting uranium fuel and building up stores of radioactive waste. The application of fast fusion neutrons can convert the huge stockpiles of depleted uranium into fresh fuel, and can help reduce waste problems by transmutation.

\subsection{The scope of the CRP}

Several options of a fusion neutron source have been considered in various member states. Neutron sources based on conventional (large) aspect ratio tokamaks have been proposed in the USA, China and the Russian Federation. They either aim at pulsed operations (Ignitor, FDF) or require the use of superconducting magnets (DEMO-FNS) that is difficult to implement in a compact device, and such facilities typically lie between JET and ITER in size. Pre-conceptual studies on the development of steady-state CFNSs are now on-going in several Member States. The majority of these investigations are based on the spherical tokamak (ST) concept. CFNS devices based on the ST concept devices have been proposed in the USA, China, UK, Brazil, Kazakhstan and the Russian Federation.

The overall objective of the CRP on 'Conceptual development of steady-state compact fusion neutron sources' is to support the research on the development of steadystate CFNSs for scientific, technological and nuclear energy applications and promote and establish collaboration among participants, industries and institutions involved in the project. The objectives of the CRP are:

- To investigate options for steady-state CFNSs with fusion power in the range of $1-100 \mathrm{MW}$ (intensity $3.5 \times 10^{17}$ $10^{19}$ neutrons $\mathrm{s}^{-1}$ ), neutron wall loading in the range $0.1-$ $1 \mathrm{MW} \mathrm{m}^{-2}$, based on magnetic confinement approaches such as tokamaks, stellarators and mirror machines, and on dense plasma focus approach;

- To explore plasma parameter spaces for optimizing plasma performance for neutron production at fusion energy gain value $Q=0.1-1$;

- To formulate concepts for enabling technologies and associated materials: this will include magnet systems, vacuum vessel, divertor, blankets, the heating and current drive systems, the pumping, cooling and fuelling systems, the tritium plant, diagnostics, the remote handling system; and to address facility safety issues;

- To formulate a joint programme of supporting $R \& D$ necessary and relevant to the eventual development of CFNS and to bring together the stakeholders and end users (such as the nuclear energy sector including fusion and fission, the basic research sector, biology and medicine sectors) of fusion neutrons for fine tuning the specific design requirements for CFNS. 


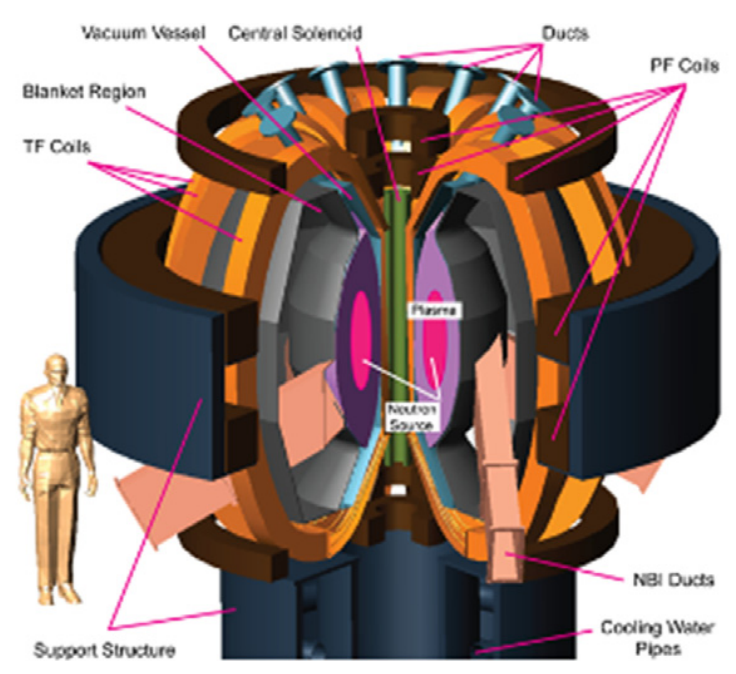

Figure 1. Cut-view of SCFNS.

This research provides concepts and conceptual designs for low and high power CFNS; determines operational domains with optimized plasma performance; produce results of joint activities on design, simulations and experiments pertinent to the development of a scientific and technological base for CFNS, give description of validated technologies and comprehensive safety analysis for the proposed CFNSs.

\subsection{Tokamak-based CFNSs}

2.2.1. Applicability of representative concepts for low power compact steady-state fusion neutron sources up to $10 \mathrm{MW}$ : super CFNSs. In a very compact ST conditions for steadystate operations require only 5-10 MW of neutral beam heating power (auxiliary plasma heating), and the wall and divertor thermal and neutron loads are within the range that can be accepted utilizing ITER technologies. Recent studies of a super CFNSs are particularly relevant. Kuteev et al [2], (figure 1), provide an example of a conceptual design of such a SCFNS. These studies specifically address the need for a small facility developing up to $10 \mathrm{MW}$ of fusion power whilst requiring total auxiliary heating and current drive power $<15 \mathrm{MW}$ and total power consumption $<50 \mathrm{MW}$. They reevaluate the smallest $\left(R_{0} \sim 0.5 \mathrm{~m}\right)$ range under quite feasible conditions: $I_{\mathrm{p}} \sim 3 \mathrm{MA}, B_{t} \sim 1.35 \mathrm{~T}$ with a neutron load below $1 \mathrm{MW} \mathrm{m}^{-2}$. Importantly for a first pilot device, the cost of building is estimated at less than 250 M USD. In [3] results of more detailed studies are presented for a device with $R_{0}=0.5 \mathrm{~m}, a=0.3 \mathrm{~m}, k=2.75, I_{\mathrm{p}}=1.5 \mathrm{MA}, B_{t}=1.5 \mathrm{~T}$, $P_{\mathrm{NBI}}=6 \mathrm{MW}, P_{\text {fus }}=1-2 \mathrm{MW}$.

\subsubsection{Applicability of representative concepts for high power} compact steady-state fusion neutron sources above $10 \mathrm{MW}$.

2.2.2.1. Texas CFNS [4]. The University of Texas CFNS is designed, primarily, as a driver (neutron supplier) for a fusion-fission hybrid (Hybrid). Driven by a copious source of fusion neutrons, the Hybrid can open qualitatively 'new' and efficient fuel cycles that may not be easily accessible to fission only methods. Through these new cycles, the Hybrid can help rid the nuclear industry of the three cardinal drawbacks associated with nuclear power - the accumulation of very long lived hazardous waste, proliferation concerns and future fuel scarcity. For these applications, which require fusion neutrons to play a major role as transmutation agents, the neutron source has to be relatively intense. The University of Texas group has conducted detailed pre-conceptual studies on neutron sources ranging from $50-400 \mathrm{MW}$ of fusion power. To make the notion of a highly intense but compact neutron source feasible, a new magnetic geometry called the super-X-divertor (SXD) was invented to withstand enormous heat and particle fluxes peculiar to all high power density fusion sources. The machine and plasmas parameters for a $100 \mathrm{MW}$, relatively light-weight, replaceable and modular, water-cooled $\mathrm{Cu}-\mathrm{Al} \mathrm{ST}$ tokamak, are: $A=1.8, R_{0}=1.4 \mathrm{~m}, k=3, \beta_{t}=30.9 \%, \mathrm{H}_{98}=1.18$, $\langle T\rangle=14.4 \mathrm{keV}, n_{\mathrm{e}}=1.44 \times 10^{20} \mathrm{~m}^{-3}$, Greenwald number $=$ 0.22, CD power $=52.5 \mathrm{MW}, I_{\mathrm{p}}=12.4 \mathrm{MA}$, bootstrap fraction $=0.35$, neutron wall loading $\sim 1.2 \mathrm{MW} \mathrm{m}^{-2}, B_{t}=2.34 \mathrm{~T}$, heating loading on the wall (with $50 \%$ heat load on SXD) $\sim 0.4 \mathrm{MW} \mathrm{m}^{-2}$. The aspect ratio $R_{0} / a=1.8$ was chosen to allow about $10 \mathrm{~cm}$ shielding for the center post and the CFNS is designed as a fully replaceable unit (that can be taken in and out of a surrounding fission blanket) - to be replaced every 1$1 / 2$ to $21 / 2$ years - to beat the very severe material constraint due to $14 \mathrm{MeV}$ fusion neutrons.

2.2.2.2. FDS-ST [5]. The FDS Team, China, proposed a ST for nuclear waste transmutation and spent fuel burning with $R_{0}=1.4 \mathrm{~m}, A=1.4, k=2.5, B_{t}=2.5 \mathrm{~T}, I_{\mathrm{p}}=9.2 \mathrm{MA}, n_{\mathrm{e}}=$ $1.1 \times 10^{20} \mathrm{~m}^{-3}$, bootstrap current fraction fbs $=0.81$, heating power $P_{\mathrm{NB}}=19 \mathrm{MW}$ and wall load $P_{\text {wall }}=1 \mathrm{MW} \mathrm{m}^{-2}$. This design with an aspect ratio near the lower limit (due to limited space in the central post) requires an unshielded centre conductor post (CCP) as a part of the toroidal field magnet. The outboard was designed as a subcritical system with a high multiplication of energy due to fission reaction in order to achieve highly economical operation. This can compensate the large fraction of recirculating power in a ST, mitigate the requirement for the neutron wall loading and thus reduce the irradiation on the first wall (FW). The CCP in the ST reactor will stand severe neutron irradiation and receive high nuclear heating power. Consequently, it is needed to be replaced after a certain years' operation. Four conventional and innovative CCP concepts, i.e. water-cooled copper (water-Cu), liquid Li self-cooled ( $\mathrm{Li}-\mathrm{SC}$ ), water-cooled $\mathrm{Li}$ (water-Li) and liquid metal blanketed copper ( $\mathrm{LM}-\mathrm{Cu}) \mathrm{CCPs}$ were investigated.

2.2.2.3. DEMO-FNS [6]. Development of a fusion neutron source DEMO-FNS for demonstrating energy valuable hybrid technologies based on a conventional tokamak with the power of deuterium/tritium fusion up to $50 \mathrm{MW}$ has been started at Kurchatov Institute, Russia. The design is aimed at the device providing steady state operation with the device life time up to 5000 operation hours with $30 \%$ availability, tritium consumption $\sim 700 \mathrm{~g} / \mathrm{y}$, the neutron flux of $\sim 0.2 \mathrm{MW} \mathrm{m}^{-2}$ and the fluence of $\sim 2 \mathrm{MW}-\mathrm{y}$ with the blanket area of $\sim 100 \mathrm{~m}^{2}$. This device should be capable of testing materials and components in the fusion neutron spectra to support the DEMO program and of developing hybrid technologies for transmutations and production of fissile nuclides. In addition this device with electric power consumption $<200 \mathrm{MW}$ is 


\begin{tabular}{|c|c|}
\hline $\mathrm{R}, \mathrm{m}$ & 2.5 \\
\hline$R / a$ & 2.5 \\
\hline$\kappa$ & 2.1 \\
\hline$\delta$ & 0.5 \\
\hline$I_{p}, M A$ & 5.0 \\
\hline $\mathrm{B}_{\mathrm{T}} \mathrm{T}$ & 5.0 \\
\hline $\mathrm{n}, 10^{20} \mathrm{~m}^{-3}$ & 1.0 \\
\hline$P_{n t n} / S, M W / m^{2}$ & 0.2 \\
\hline $\mathrm{E}_{\mathrm{b}}, \mathrm{keV}$ & 500 \\
\hline$P_{b}, M W$ & 35 \\
\hline Angle NBI, deg & 20 \\
\hline$P_{E C}, M W$ & 6 \\
\hline H-factor & 1.0 \\
\hline$\beta_{N}$ & $<3$ \\
\hline $\mathbf{f}_{\text {non-ind }}$ & 1.0 \\
\hline$P_{\text {diss. }}$ TF. MW & 10 \\
\hline$P_{\text {diss }}$, PF, MW & 5.0 \\
\hline $\mathrm{S}_{\text {wall }} \cdot \mathrm{m} 2$ & 162 \\
\hline $\mathrm{V}_{\mathrm{pl}} \cdot \mathrm{m} 3$ & 103 \\
\hline
\end{tabular}

\section{Parameters of} DEMO-FNS

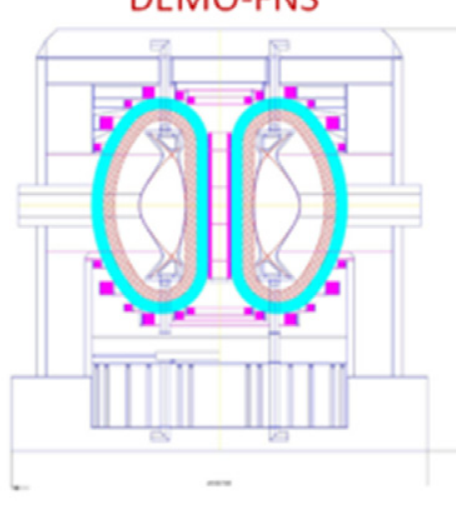

Demonstration

-Tritium breeding

- MA incineration

- Fissile nuclide production

- Destructiony of long life

radionuclides

- Heat transfer

B.V. Kuteev, 6 December, 2013, FNS CRP meeting

Figure 2. Main parameters and a cross-view of DEMO-FNS.

aimed to demonstrate the closed energy cycle using fission power up to $500 \mathrm{MW}$ from fissile nuclides located in the blanket. These demands mainly determine the size of the device setting the necessary blanket area. The table of its main parameters and a cross-view are shown in figure 2.

\subsection{Non-tokamak steady-state fusion neutron sources}

The need to drive the toroidal plasma current in axisymmetric toroidal devices is a serious obstacle to finally develop a steadystate fusion neutron source in such devices. In particular, the power required to drive such current could turn out to be too high for a reactor realization. The need for a current drive is removed in open systems (mirror machines etc) or toroidal systems with a rotational transform (stellarator or more preferably a stellarator-mirror systems where the fusion neutron generation is localized to a mirror machine section of the toroidal device), allowing steady-state operations. Open geometry provides certain flexibility in technical solutions (for divertor, diagnostics, etc), where axisymmetric mirror machines have the simplest magnet. In recent years, a rather dramatic improvement in plasma performance in axisymmetric mirrors have been demonstrated (in the single cell GDT and the multimirror GOL-3 experiments at the Budker Institute) [25, 26]. Quadrupolar fields are considered in the straight field line mirror (SFLM) concept for gross plasma stability. A remaining concern is the longitudinal plasma loss in open systems. However, experiments on GDT device in Novosibirsk [27] have already demonstrated, in agreement with theory, that strong expansion of the magnetic field in the end tanks beyond the mirrors inhibited the high heat losses connected to thermal electron conduction. According to the experiments, the end losses are determined by the plasma exhaust through the mirrors, i.e. by the plasma directed velocity and plasma density in the mirrors, as predicted by theory. The stellarator-mirror concept eliminates the axial heat losses completely, although there is a certain price in added complexity.

One of the major advantages of mirrors is the high achievable beta value, which can be as high as $60 \%$. This has been successfully demonstrated in the GDT experiment. Also, since the high neutron flux can be localized in the certain regions by skew injection of the neutral beams, it allows for flexible arrangement with sensitive equipment outside regions of high neutron flux. Therefore, a sufficient neutron shielding can be arranged, and the device components can operate without replacement of systems for decades. Power loads on the expander plates (which play the role of 'divertor plates') and the load on the FW are some other examples where the open geometry provides solutions.

Plasma confinement (end loss in open systems, neoclassical banana effects for quadrupolar mirrors and radial loss in stellarators) are of some concern. In many other aspects, the challenges seem less severe than for tokamaks, but insufficient plasma confinement is a real threat. Material load seems tolerable. Plasma heating in steady state could be a challenge, but ion cyclotron heating (and probably neutral beam injection (NBI), possibly also electron cyclotron resonance heating (ECRH)) are candidates.

There is also an interest for smaller neutron sources, where an intense pulsed 'neutron flash' could have certain applications. The dense plasma focus is an example of such a compact neutron source [28]. Plasma focus devices cannot be treated as a steady state machines because of their intrinsic pulsed nature, but they can operate in repetitive regimes. This capability has been already demonstrated on the small scale (few kJ machines). Present day poloidal field (PF) devices of 
the $1 \mathrm{MJ}$ level (energy stored in the capacitor bank) are capable to emit up to $Y_{n} \sim 10^{12}$ of D-D $(\sim 2.5 \mathrm{MeV})$ and $Y_{n} \sim 10^{14}$ (14 MeV DT) neutrons per discharge [29].

Experimental scaling laws for plasma focus are promising, namely the neutron yield for discharge scales with the stored energy as $Y_{n} \sim E^{2}$. It seems that with the present technology it is possible to build an efficient neutron source generating $10^{17}$ neutrons $\mathrm{s}^{-1}$. There is a renewal of interest in research aimed at application of PF as the intense neutron sources [29]. For instance a line of high energy PF devices has been constructed at the National Security Technologies, LLC, under contract with US Department of Energy. The aim of the Project is to work out a conceptual design of the intense neutron source $\left(\sim 10^{17}\right.$ neutrons $\left.\mathrm{s}^{-1}\right)$ based on the repetitive plasmafocus principle for various advanced applications.

\section{CRP F1.30.14 'Utilization of a network of small magnetic confinement fusion devices for mainstream fusion research'}

The overall objective of CRP on 'Utilization of a network of small magnetic confinement fusion devices for mainstream fusion research' is to contribute to streamlining the inputs of results of studies on small magnetic confinement fusion devices to mainstream fusion research by establishing a network of cooperation enabling coordinated investigations of topics of relevance to physics, diagnostics and technology issues of next step fusion devices such as ITER and DEMO. This is provided by:

- Establishment of a network of small magnetic confinement fusion devices and utilization of the network to perform joint (IAEA JEs) and comparative experiments;

- Utilization of the network to support technology development, e.g. novel diagnostics, materials and techniques, development of prototypes of applicable fusion-relevant simulation and communication tools and modelling analysis tools;

- Supporting training and education activities for members of the network.

Results of JEs and following independent activities on small Fusion devices utilize the flexibility of these devices, their experimental programmes, well developed diagnostics and the high skill of their personnel. The contribution of small fusion devices to the mainstream fusion research can be enhanced through coordinated planning and JEs.

JEs in the framework of the IAEA CRP on 'Joint research using small tokamaks' have been carried out on tokamaks CASTOR at IPP Prague, Czech Republic, tokamak T-10 at RRC 'Kurchatov Institute', Moscow, Russia, tokamak ISTTOK at IST, Lisbon, Portugal, TCABR tokamak at the University of São Paulo, Brazil in 2005-2009, and under the new CRP on 'Utilization of a network of small magnetic confinement fusion devices for mainstream fusion research' on COMPASS and GOLEM tokamaks in 2012 and 2013. In the first JEs, experimental programs were aimed to diagnose and characterize the core and the edge plasma turbulence in a tokamak in order to investigate correlations between the occurrence of transport barriers, improved confinement, electric fields and electrostatic turbulence using advanced diagnostics with high spatial and temporal resolution. In recent JEs, studies have been extended to characterization of the pedestal in ohmic and NB heated H-mode discharges, $q$-dependence and non-linear evolution of the NBI-induced Alfvén eigenmodes (AEs), edge plasma studies and microwave emission, relation between halo currents and $3 \mathrm{D}$ asymmetries of $I_{\mathrm{p}}$ during disruptions, evaluation of the parallel electron power flux density using Langmuir and Ball-pen probes, investigation of RF pre-ionization and investigation of the use of high-temperature superconductors (HTS) in PF coils of a tokamak. These JEs have also clearly demonstrated that small fusion devices are suitable for broad international cooperation to conduct dedicated joint research programmes.

\subsection{Contribution to the mainstream fusion research}

Research on small fusion devices is typically restricted to specific areas and education/training purposes. However, several small devices, i.e. Compass-D, Alcator C-Mod, T-10, Globus-M, START, RTP, TEXT, TJ-2, Uragan-2M, Uragan-3 and others have (or had) extended research programmes, wellequipped with sophisticated diagnostics and powerful heating systems. Figure 3 shows results from very advanced Thomson scattering diagnostics on COMPASS, IPP-AS Prague [30, 31], and comparison of Thompson scattering (TS) measurements with very high core and edge resolution with reflectometry and Langmuir probes (LPs). Similar diagnostics on C-Mod, T-10 and Globus-M allows detailed investigation of the tokamak transport. Results from Compass-D, C-Mod and START made significant contributions to the ITER confinement database.

Results from small fusion devices obtained during previous JEs and from individual co-ordinated activities are well described in [7-9]. Here we present only some selected examples of recent results of significant importance to the mainstream fusion research.

Characterization of scape-off-layer (SOL) and off-SOL plasmas is very important for the power balance at the plasma edge and wall and divertor loads. It is well known that peripheral plasmas of small and big tokamaks have many common features. The electron distributions in the SOL plasma of Alcator-C Mod, DIII-D, and Tokamak-de-Varennes have been shown to be non-Maxwellian for both attached and detached operation regimes.

The non-Maxwellian tail of the electron energy distribution function (EEDF) strongly affects plasma diagnostics, parallel plasma heat fluxes and plasma-neutral and plasmaimpurity interactions. An elevated non-Maxwellian tail into the plate results in an overestimate of the temperature estimation by divertor Langmuir probes by a factor of $2-6$, using classical probe techniques $[10,14]$.

The aim of the experiment was to investigate the influence of the non-Maxwellian EEDF on SOL plasma properties by evaluating the plasma potential and the real EEDF in the COMPASS tokamak (radial measurements by horizontal reciprocating probes and 39 divertor probes) during L- and $\mathrm{H}$-mode in deuterium plasma. Figure 4 shows results of measurements of the EEDF obtained using first-derivative method [10] during JE on CASTOR tokamak, IPP Prague. It clearly shows the bi-Maxwellian nature of the EEDF. Similar results were obtained on COMPASS tokamak in Prague during 

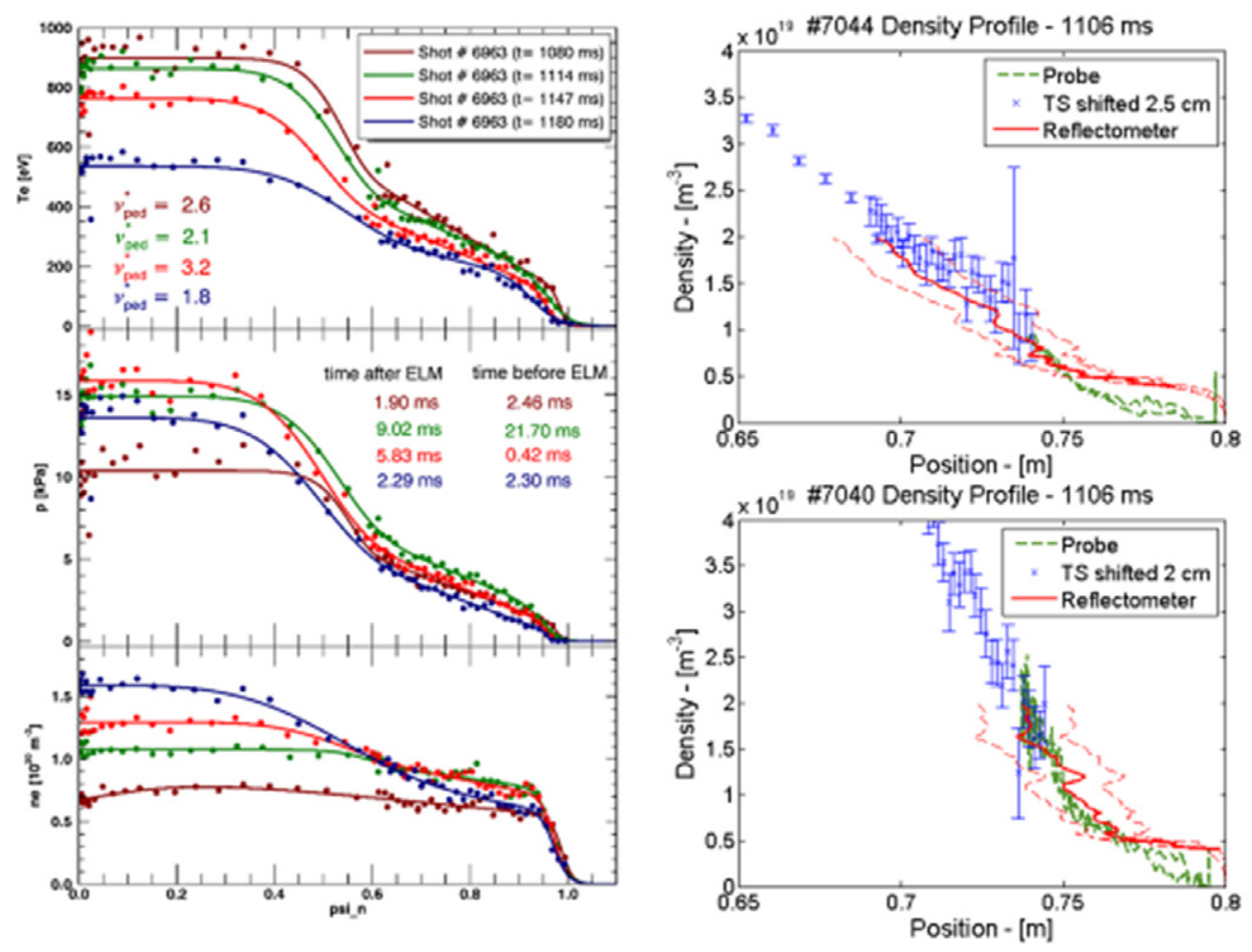

Figure 3. TS results and comparison with LP and reflectometer on COMPASS-D, Prague.

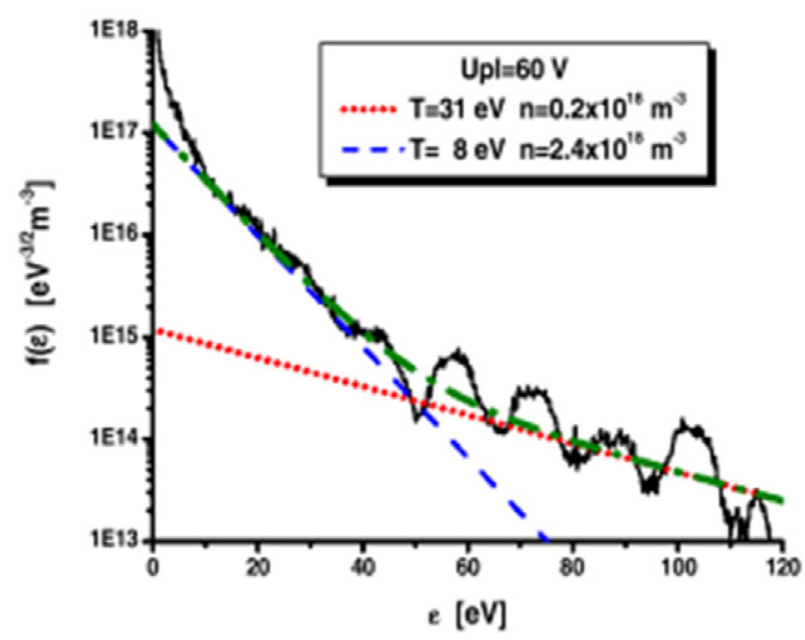

Figure 4. EEDF measured with LPs at the edge of CASTOR tokamak.

the 6th JE in 2013. Figure 5 shows the radial distribution of the electron temperatures and densities in the vicinity of the last closed flux surface (LCFS) and in far SOL. Both temperature and density measurements show bi-Maxwellian features in the vicinity of the LCFS while in the far SOL the EEDF is Maxwellian.

Figure 6 presents contribution of the COMPASS, Prague, tokamak results into the database of the energy loss in energy localized modes (ELMs) depending on the pedestal electron collisionality. Utilization of NBI available on COMPASS will result in the extension of the present observations to lower collisionality.
Another area of the CRP and JE research was AE studies. Instabilities driven by energetic ions have been observed in many fusion devices of various size, i.e in tokamaks [11] and stellarators $[12,13]$. The induced modes, AEs are driven by the energy transfer from energetic ions to the waves. Energetic electrons may also excite quasi-coherent modes under some conditions $[14,15]$.

During JE6, studies of AE modes on COMPASS tokamak have been performed in the plasma heated by the $40 \mathrm{keV}$ neutral beam. The port-through power $P_{\mathrm{NBI}}<0.3 \mathrm{MW}$, beam diameter $5 \mathrm{~cm}$, pulse length $<0.3 \mathrm{~s}$. The beam velocity is $V_{\mathrm{b}}=\sqrt{ } 2 E_{\mathrm{b}} / m_{\mathrm{D}}=2 \times 10^{6} \mathrm{~m} \mathrm{~s}^{-1}$, so the beam was subAlfvénic $V_{\mathrm{b}}<V_{\mathrm{A}}$ at densities $n_{\mathrm{e}}<8 \times 10^{19} \mathrm{~m}^{-3}$. Here $V_{\mathrm{A}}$ is Alfvén velocity

$$
V_{\mathrm{A}}=\frac{B}{\sqrt{\mu_{0} \sum_{i} n_{i} m_{i}}},
$$

$n_{i}$ is a density of the particles of type $i, m_{i}$ is the particle mass.

The Alfven-like quasicoherent modes were typically observed within the frequency range of $50<f<250 \mathrm{kHz}$, figure 7. Some of the modes are visible only on the low field side (LFS), right, and not visible on the high field side (HFS), left plot. Consequently, these modes have clear ballooning character with the typical frequency in the range of $50<f<$ $150 \mathrm{kHz}$, while modes with antiballooning character have the typical frequency range of $150<f<250 \mathrm{kHz}$, left.

Both ballooning and antiballooning modes coexist, as shown in the figure 7. The antiballooning modes have higher frequencies and wider frequency interval than the ballooning ones. The cross-phase analysis gives preliminary estimate of the poloidal mode number $m=5.5 \pm 1$ for ballooning 

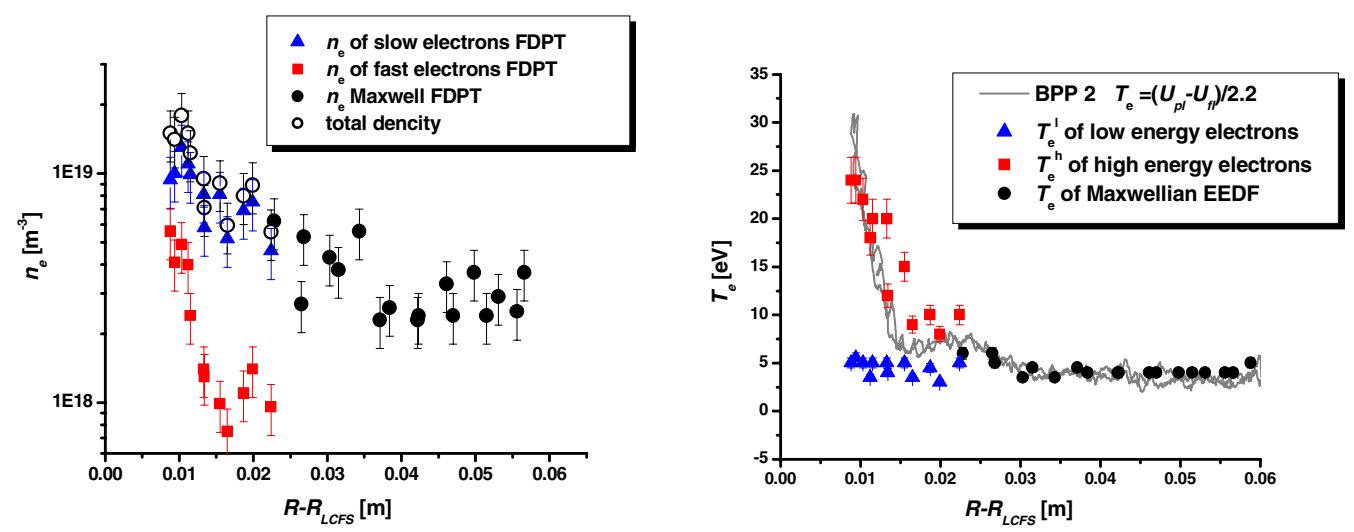

Figure 5. Electron density, left, and temperature, right, in the SOL of the COMPASS tokamak.

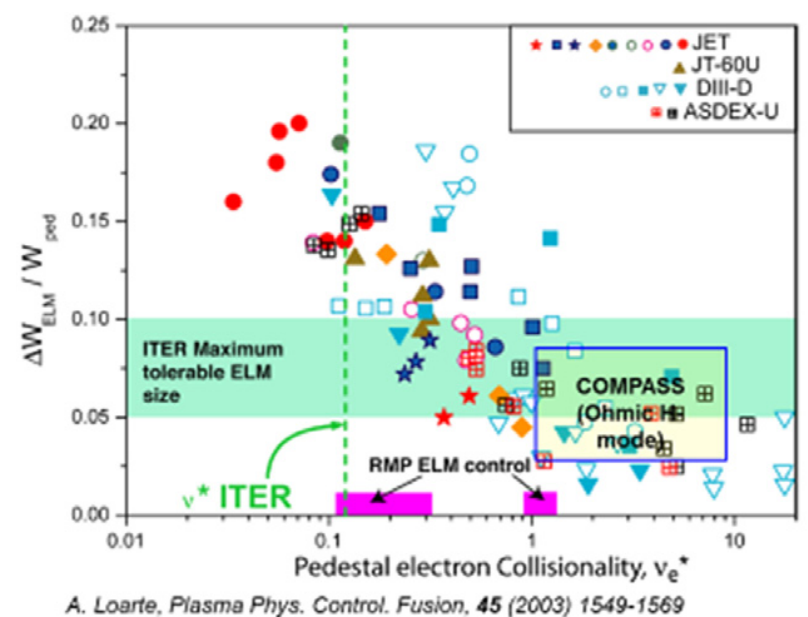

Figure 6. Energy loss in the ELM depending on the collisionality in various tokamaks.

modes. For antiballooning modes, the exact $m$ detection was not possible due to diagnostic limitations, the estimates gives $m<8$. Both ballooning and antiballooning modes seem to rotate in electron diamagnetic drift direction. It is important to mention that the modes were observed only in $\mathrm{H}$-mode phase of the discharge [16].

Small tokamaks allow extensive studies in areas where such experiments on big tokamaks are complicated and risky. An example is the disruption studies. Plasma current asymmetries during disruptions have been studied during JE on COMPASS and results have been compared with JET results [17].

\subsection{Development of fusion technologies}

One of the major risks on our path to fusion power is connected with the desire to rely on fast progress in the development of materials, both for the FW/divertor and for magnets, and on the current drive technologies. Small fusion devices can provide significant a contribution to resolving some of the relevant issues.

The importance of investigation of capabilities and properties of HTS for application in magnets of the future Fusion devices is well defined in the EuroFusion roadmap [1]. The first application of HTS in PF magnets has been demonstrated on a small tokamak GOLEM at the Technical University of Prague as a contribution to the CRP activity work plan [13]. Figure 8, left, shows plasma in GOLEM tokamak supported with HTS PF coils at LN temperature.

Within the CRP programme, experiments have been performed on two small tokamaks, ST25 and HTS-ST tokamak, figure 9 . Both devices have a circular plasma crosssection, $R / a=0.25 / 0.12 \mathrm{~cm}, I_{\mathrm{p}}$ up to $10 \mathrm{kA}, \mathrm{TF}=0.1-0.3 \mathrm{~T}$ in ST25, left, and 0.1-1 T in fully-HTS tokamak ST-HTS, right. These tokamaks use various RF power sources (EBW, ICRH) to provide fully non-inductive current derive. Experience gained from operation of the ST25 and the first fully-HTS tokamak ST-HTS will contribute to the design of the next step tokamaks (i.e. DEMO) magnets and also are providing physics results on the EBW current drive as they are equipped with different RF launch geometries (steerable low- and the unique up to date high-field launch of RF power from two $2.45 \mathrm{GHz}, 3 \mathrm{~kW}$ magnetrons). RF-driven plasma with pulse duration of $25 \mathrm{~s}$ are routinely available on the ST25 tokamak, with $\mathrm{Cu}$ TF magnets powered by the Maxwell supercapacitors power supplies. The full-HTS tokamak HTS-ST has already performed $24 \mathrm{~h}$ operations, figure 10 , which demonstrated the first continues operation of a tokamak in He. Several spikes in the waveforms correspond to temporary loss of the RF coupling to the plasma, and can be avoided. Video of the discharge can be found at www.tokamakenergy.co.uk. Formation of the closed flux surfaces has to be confirmed in the future studies in hydrogen with the increased RF power. In these experiments, $\sim 1 \mathrm{~kW}$ of $13 \mathrm{MHz}$ RF power has been injected, see figure 9, right, with very low level of the reflected power. Tests of $\mathrm{Li}$ conditioning of the plasma wall will be carried out in 2014.

Studies of the electron cyclotron resonance (ECR) preionization system have been performed during 6th JE on GOLEM using $2.45 \mathrm{GHz} 1 \mathrm{~kW}$ magnetron from a standard microwave oven, figure 8, right. The use of commercial magnetrons allowed the pre-ionization system to be very cheap and so affordable for small tokamaks.

\subsection{Education and training}

The experimental work on small tokamaks is very appropriate for educating students, for the scientific activities of postgraduate students, provides good experimental basis for $\mathrm{PhD}$ research. Small tokamaks are very suitable for training of 

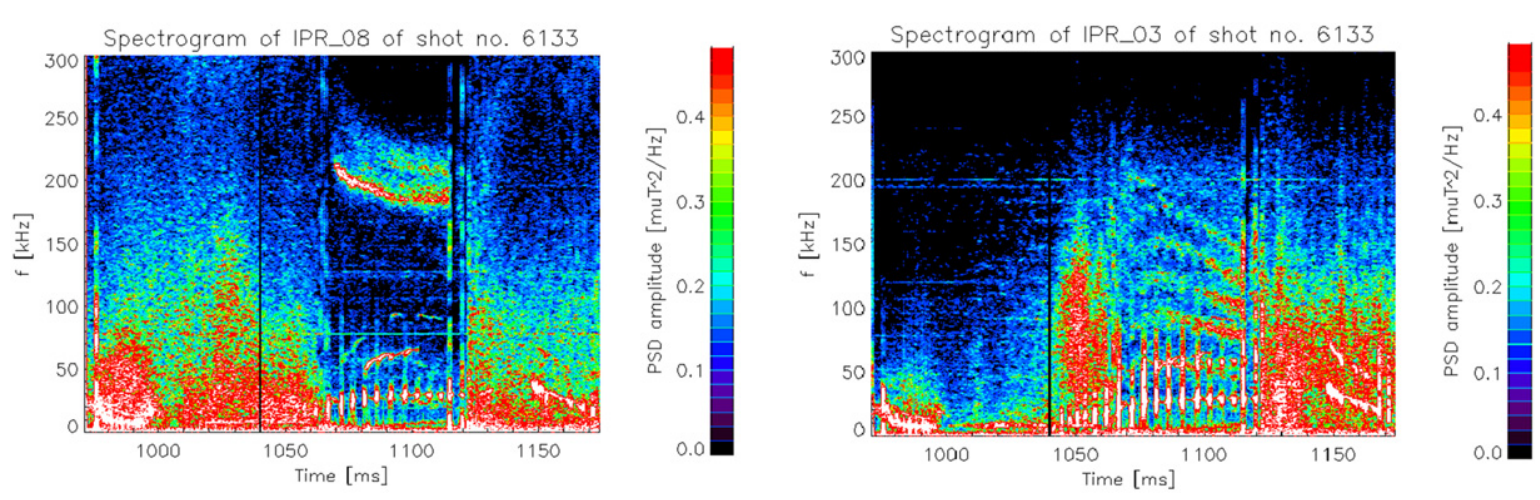

Figure 7. Power spectrogram of the magnetic probes signals taken at HFS (left), and LFS (right). The AEs appears during the H-mode phase of the discharge.
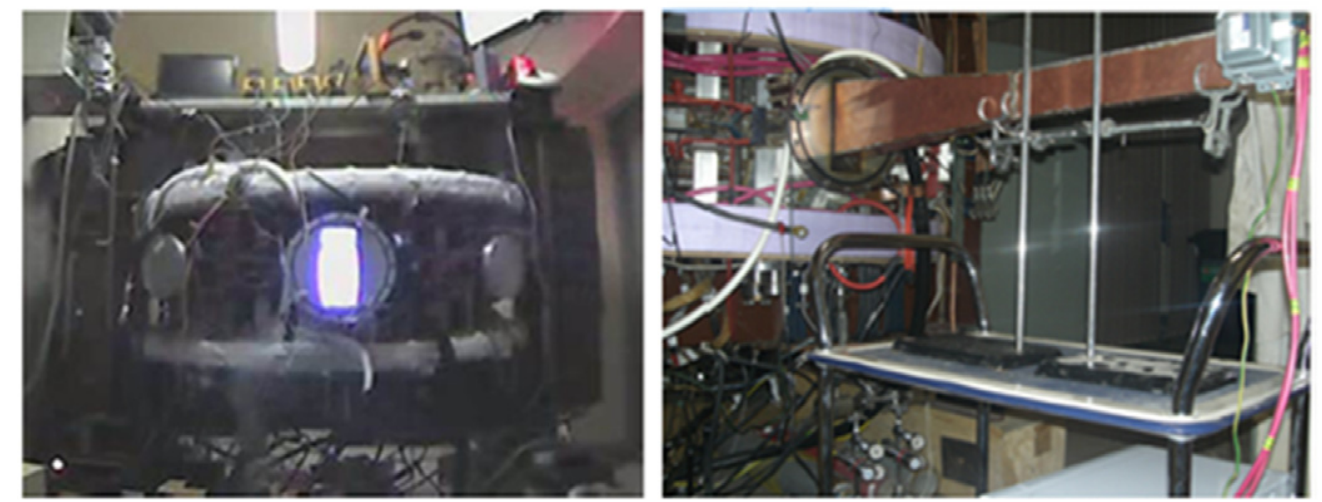

Figure 8. Plasma in GOLEM tokamak supported by HTS PF coils, left. Right—EC pre-ionization tests on GOLEM.
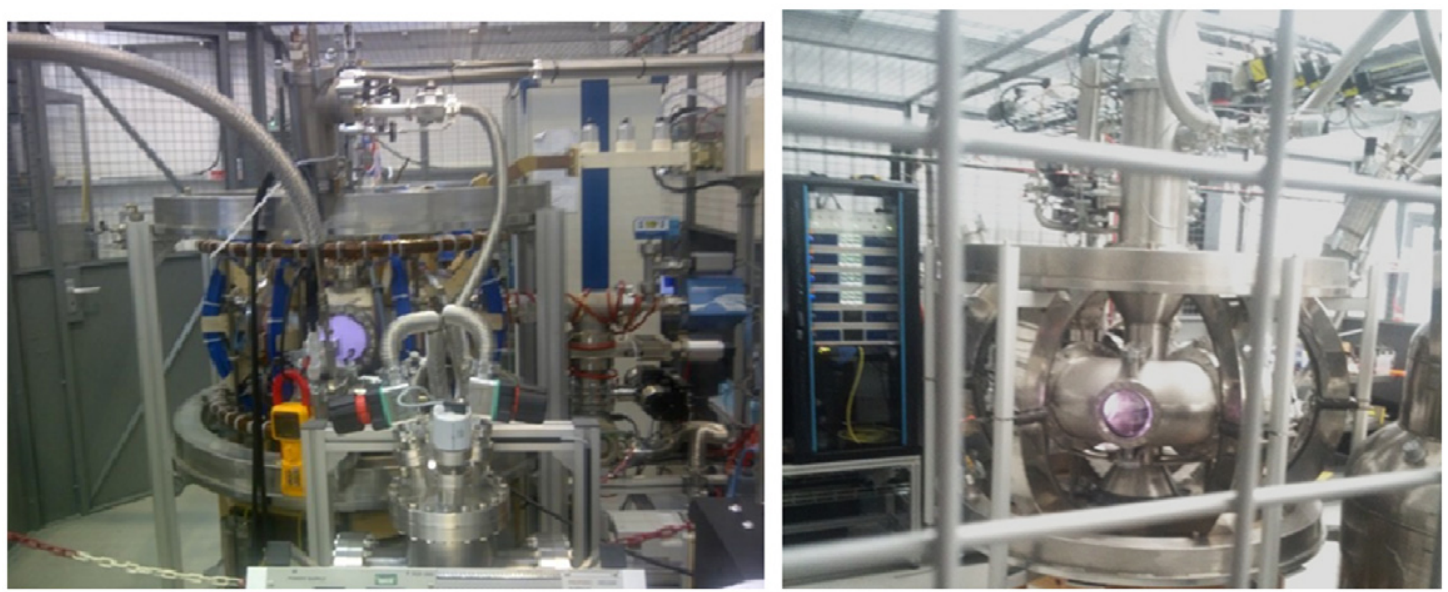

Figure 9. ST25 tokamak with HTS PF coils, left. First plasma in full-HTS tokamak, right.

personnel for larger tokamaks. An opportunity to participate in International activities in Fusion opens the door for more member states to join the research efforts and contribute to the success of the development of fusion. Educational activities within this CRP are intended to take advantage of some of the existing offers, including international schools, workshops and training courses.

Scientific exchange within the network will be supported on the basis of in-kind contributions, with special emphasis on young experts from developing countries being interested in working at small fusion devices worldwide. Moreover, young scientists from the network should develop interest in the specific needs of the laboratories in developing countries and be encouraged to undertake scientific stays in these countries, e.g. under the IAEA technical co-operation agreements. Figure 11 shows some of tokamaks operating in developing countries participating in the IAEA CRP.

Educational activities within IAEA CRPs are increasing, for example two schools have been recently organized at IPP Prague: EMTRAIC School in December 2013 and SUMTRAIC Summer School in August 2014 Several International schools have been organized jointly by IAEA 

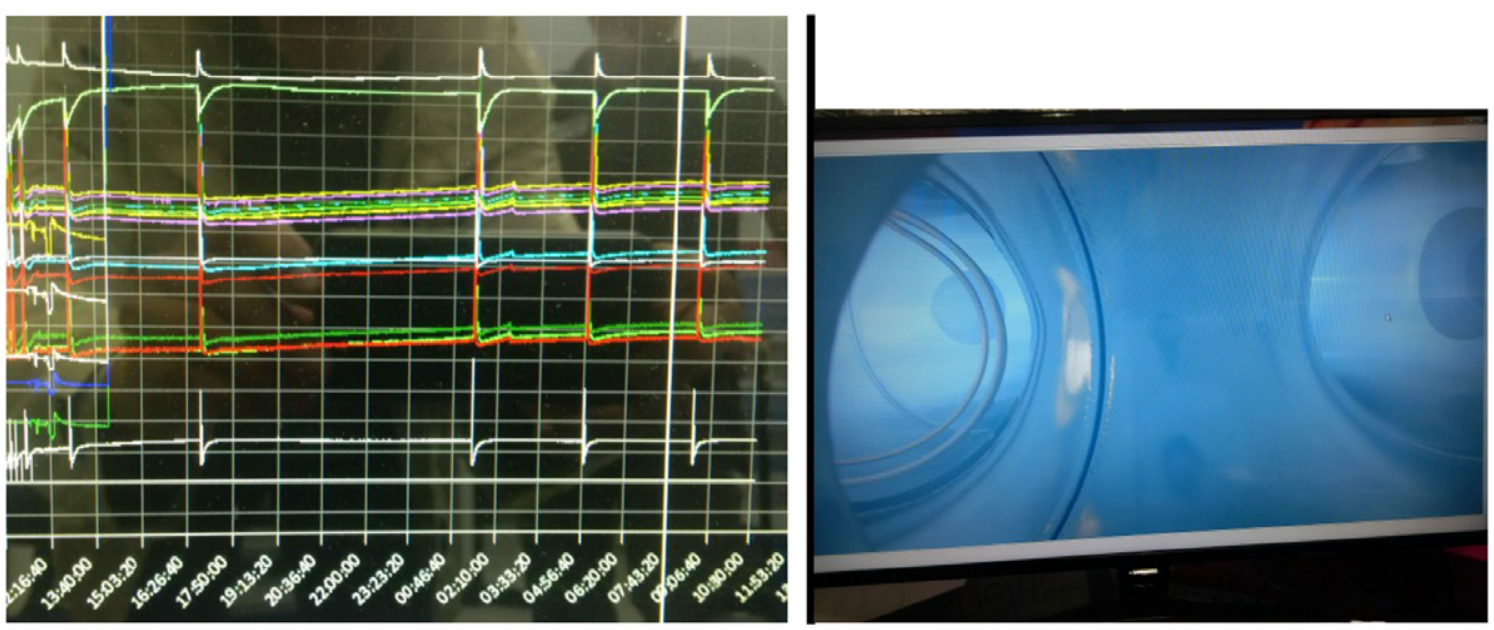

Figure 10. Left: typical waveforms from continues plasma discharge in HTS tokamak. Top two: reflected and injected RF power $(<1 \mathrm{~kW}$ in this case), next-temperatures in different HTS TF coils. Axis $X$ - in hours, beginning of the discharge at $\sim 11: 30$ am. Right: typical discharge with RF antenna seen at the left.
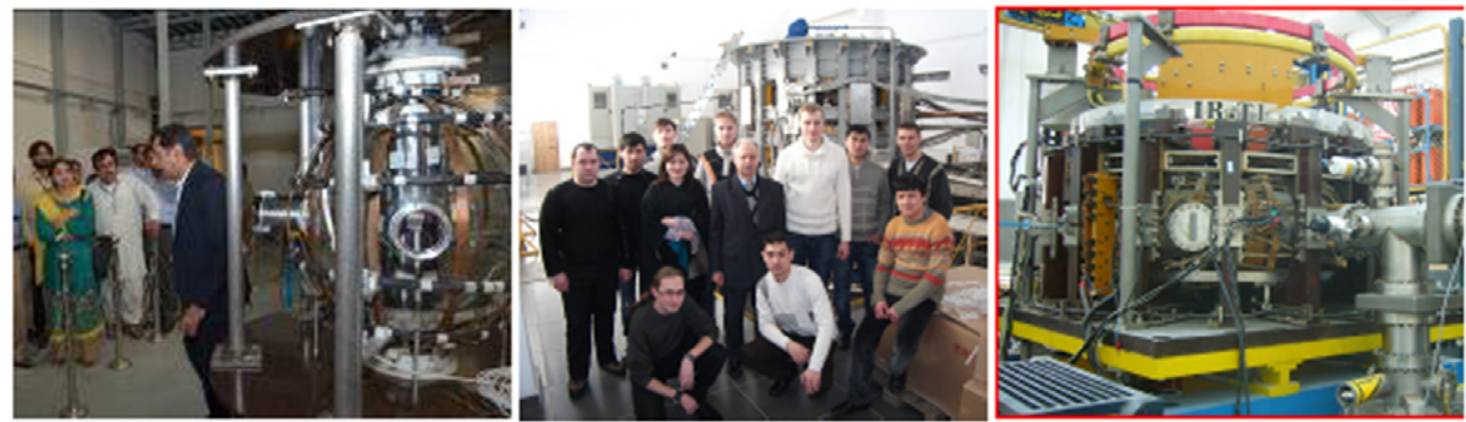

Figure 11. GLAST tokamak, Pakistan, KTM, Kazakhstan, and IR-T1 of Iran.

and ICTP. Erasmus Mundus, FusNet and other activities are also contributing to CRP activities, for example during recent Fusion Energy course for DTU students remote experiments on two small tokamaks participating in the CRP, ST25 [18] and GOLEM [32], have been performed. Only JEs and SUMTRAIC/EMTRAIC and ICTP schools had 176 students participating, and the number of $\mathrm{PhD}$ students involved in the CRP activities exceeds 60.

\section{Conclusions}

IAEA Coordinated Research Projects are already paying visible dividends and have resulted in a substantial number of joint presentations and publications. Combined and coordinated efforts within a network of small fusion devices enhance the contribution of small devices to mainstream nuclear fusion R\&D. The IAEA Joint Experiments have clearly demonstrated that small tokamaks are suitable and important for broad international cooperation, providing the necessary environment and manpower to conduct dedicated joint research programs. In this paper we are presenting results of recent activities, selecting the most important and novel, i.e. first application of HTS in tokamak magnets on GOLEM and first results from all-HTS tokamak; observation of new MHD modes on COMPASS and bi-Maxwellian EEDF at the edge of COMPASS and CASTOR plasmas, etc. Practical advice and assistance via IAEA help further integration of activities on small fusion devices with the national programs, ITER and other international projects. Joint research on future devices, i.e. compact fusion neutron source under the IAEA CRP, helps not only to coordinate activities and provide exchange of information on the design of CFNS, but links research on tokamaks with that on open traps, stellarators, dense plasma focus and other fusion devices.

\section{Acknowledgments}

Most of experimental data were obtained during joint experiments performed in the framework of IAEA CRP 'Utilisation of a network of small magnetic confinement fusion devices for mainstream fusion research, 2011-2015'. Authors are grateful to JEs hosting COMPASS team for kind hospitality, enthusiastic work and friendly collaboration. COMPASS data analysis and modelling performed by the Kurchatov team (section 3.1) was carried out within the Russian Scientific Foundation, project 14-22-00193.

\section{References}

[1] Donné A.J.H. 2014 JET Science Meeting (15 September 2014)

[2] Kuteev B.V. et al 2010 Intense fusion neutron sources Plasma Phys. Rep. 36281

[3] Kuteev B.V. et al 2011 Steady-state operation in compact tokamaks with copper coils Nucl. Fusion 51073013 
[4] Kotschenreuther M. et al 2009 Fusion-fission transmutation schemes-efficient destruction of nuclear waste Fusion Eng. Des. 8483

[5] Wu Y. and The FDS Team 2006 Conceptual design activities of FDS series fusion power plants in China Fusion Eng. Des. 812713

[6] Kuteev B.V. et al 2014 Development of DEMO-FNS tokamak for fusion and hybrid technologies Proc. 25th Int. Conf. on Fusion Energy (St. Petersburg, Russia, October 2014) FIP/P7-24 www-naweb.iaea.org/napc/physics/ FEC/FEC2014/index.htm

[7] Gryaznevich M.P. et al 2005 Joint research using small tokamaks Nucl. Fusion 45 S245

[8] van Oost G. et al 2007 Joint experiments on small tokamaks: edge plasma studies on CASTOR Nucl. Fusion 47378

[9] Gryaznevich M. et al 2009 Results of joint experiments and other IAEA activities on research using small tokamaks Nucl. Fusion 49104026

[10] Popov T. et al 2009 Plasma Phys. Control. Fusion 51065014

[11] Sharapov S. et al 2004 Phys. Rev. Lett. 93165001

[12] Melnikov A.V. et al 2010 Nucl. Fusion 50084023

[13] Jimenez-Gomes R. et al 2011 Nucl. Fusion 51033001

[14] Melnikov A.V. et al 2011 Plasma Fusion Res. 62402030

[15] Valovic M. et al 2000 Nucl. Fusion 401569

[16] Melnikov A.V. et al 2015 Plasma Phys. Control. Fusion 57065006

[17] Gerasimov S.N. et al 2014 Nucl. Fusion 54073009

[18] Gryaznevich M. et al 2013 Progress in application of high temperature superconductor in tokamak magnets Fusion Eng. Des. 881593

[19] Jassby D.L. 1977 Nucl. Fusion 17309

[20] FESAC 2003 Non-electric applications of fusion Report to FESAC http://science.energy.gov/ /media/fes/fesac/ pdf/2003/Fesac_final_non_elec_2003.pdf
[21] Bourque R.F. and Schultz K.R. 1988 Fusion applications and market evaluation (FAME) study Report GA-A18658/UCRL-21073 (San Diego, CA: GA Technologies) www.dtic.mil/dtic/tr/fulltext/u2/a243768.pdf

[22] US Department of Energy 1982 Technology spin-offs from the magnetic fusion energy program Report DOE/ER-0132 http://inis.iaea.org/search/searchsinglerecord.aspx? recordsFor $=$ SingleRecord $\& \mathrm{RN}=14721857 \#$

[23] US Department of Energy 1993 The US fusion program as a source of technology transfer www-fusion.ciemat.es/ New_Fusion/es/Fusion/documentos/Spinoff_DOE_1993.pdf

[24] Steiner D.E. et al and The ARIES Team 2000 The ARIES fusion neutron-source study UCSD-ENG-0083 http://aei.ucsd.edu/LIB/REPORT/ARIES-MISC/ FNS-final.pdf

[25] Burdakov A.V. et al 2011 Concept of fusion reactor based on multi-mirror trap Fusion Sci. Technol. 599

[26] Burdakov A.V. et al 2009 Status and prospects of GOL-3 multiple-mirror trap Fusion Sci. Technol. 5563

[27] Ivanov A.A. et al 2013 Auxiliary electron heating and plasma control in GDT device with electron beam: the results of initial experiments Fusion Sci. Technol. 63289

[28] Gribkov V.A. et al 2010 Phys. Scr. 81035502

[29] Gribkov V.A. et al 2011 Nucl. Fusion 51127001

[30] Panek R. et al 2006 Reinstallation of the COMPASS-D Tokamak in IPP ASCR Czechoslovak J. Phys. 56 B 125

[31] Bohm P. et al 2014 Edge Thomson scattering diagnostic on COMPASS tokamak Installation, calibration, operation, improvements Rev. Sci. Inst. 85 11E431

[32] Svoboda V. et al 2011 Multi-mode remote participation on the GOLEM tokamak Fusion Eng. Des. 861310 